\title{
A biased Monte Carlo technique for calculation of the density of states of polymer films
}

\author{
Tushar S. Jain and Juan J. de Pablo ${ }^{a)}$ \\ University of Wisconsin-Madison, Department of Chemical Engineering, Madison, Wisconsin 53706
}

(Received 11 December 2001; accepted 31 January 2002)

\begin{abstract}
A new Monte Carlo algorithm is implemented for simulation of the density of states of free-standing polymer films. The algorithm combines the original idea of conducting a random walk in energy space with advanced trial moves such as configurational bias and end-bridging. Excellent agreement is found between the results of this new method and those from simulations in the canonical ensemble, down to temperatures in the vicinity of the apparent glass transition. The efficiency of the new algorithm is studied as a function of the types of trial moves employed. It is found that, depending on the range of energy and density, certain localized moves fail to converge to the correct distribution of states. (C) 2002 American Institute of Physics. [DOI: 10.1063/1.1463422]
\end{abstract}

\section{INTRODUCTION}

The study of ultra-thin polymer films (on the order of molecular dimensions) has recently attracted considerable attention. Polymers in thin films exhibit properties, both dynamic and static, that can be significantly different than those of the bulk. In particular, the glass transition temperature of a polymer in a thin film geometry has been found to depend on thickness and, for supported films, on surface energy. ${ }^{1-5}$

A complete understanding of the origins of this behavior is still lacking. One of the challenges facing theory is that many of the assumptions that can be made regarding molecular structure in the bulk become questionable in nonisotropic, thin-film geometries. Molecular simulations could provide valuable insights into the structure of long chain molecules in thin films. Unfortunately, simulations of polymer thin films near the glass transition $T_{g}$ have been scarce. This can be attributed to the long relaxation times that arise near $T_{g}$, and the difficulties associated with sampling the energy landscape of a system as it approaches $T_{g}$.

One of the prevailing pictures of a glass is provided by the framework of energy landscape theory, ${ }^{6,7}$ which postulates that as the glass transition is approached, the system tends to reside in potential-energy basins separated by large barriers. Over short time scales, the system is only able to explore the vicinity of an energy minimum. The dynamics over longer time scales are dictated by transitions between different basins. As the glass transition temperature is approached, the short time motion becomes increasingly decoupled from the long time dynamics. Conventional molecular dynamics simulations are unable to generate sufficiently long trajectories near $T_{g}$ to overcome energy barriers. Conventional Monte Carlo techniques are unable to sample the system's energy landscape adequately; advanced trial moves and sophisticated methods are needed in order to generate meaningful ensemble averages. The purpose of this work is to present a technique that permits efficient simulation of the

a) Author to whom correspondence should be addressed. structure and properties of thin films down to temperatures in the proximity of the glass transition.

Recently, a novel simulation technique was proposed by Wang and Landau ${ }^{8,9}$ which samples configuration space not according to a Boltzmann weight, but according to the density of states $g(E)$ of the system of interest. This method could provide an attractive tool for simulation of near glassy systems because it effectively eliminates free-energy barriers between distinct configurations, thereby facilitating sampling of an otherwise rough energy landscape. As originally proposed, however, the so-called density-of-states (DOS) Monte Carlo method employed simple local moves to generate the density of states of an Ising lattice model. In this work, we show how that method can be modified to incorporate more advanced, biased moves which are necessary for effective sampling of polymer systems. The resulting algorithm is then applied to simulate free-standing polymer films as they undergo an apparent glass transition.

In principle, the DOS method requires that the energy range to be sampled be specified before a simulation. The range of $E$ can be arbitrarily wide in the region of interest. However, it is computationally efficient to perform the calculation over smaller sized overlapping energy windows, and to combine the results to obtain $g(E)$ over a large energy range. The density of states thus obtained can then be used to determine thermodynamic averages as a function of temperature of the system. The resolution in temperature can be as fine as desired, thereby permitting precise study of a system's properties near any point of interest (such as the glass transition temperature).

In the particular case of glass forming systems near $T_{g}$, if the size of an energy window is not larger than the energy barriers that separate energy minima, or if the trial moves are not powerful enough to tunnel through these barriers, then the random walk can only sample energies in the immediate vicinity of these minima. In this work, we have examined the effect of using different types of trial moves over energy ranges that encompass melt and glass conditions, and find that near $T_{g}$, certain types of localized, trial moves are un- 
able to converge to the correct distribution in a reasonable amount of computer time. We have implemented modified algorithms that combine more elaborate trial moves such as simple configurational bias and topological configurational bias with the method of Wang and Landau. It is shown that this new algorithm is effective for simulation of thin polymer films in the vicinity of the glass transition.

\section{METHODS}

\section{A. Algorithm}

The original implementation of the DOS method was conducted on a 2-D Ising model and, more recently, on the 2-D ten-state Potts model. ${ }^{8}$ As originally proposed, the method performs a random walk in energy space, with a probability proportional to the reciprocal of the density of states $1 / g(E)$. At the start of a simulation, the density of states is set to unity for all values of energy, i.e, $g(E)=1$ for $E_{\min }<E<E_{\max }$, where $\left[E_{\min }, E_{\max }\right]$ is the specified energy range to be studied. Trial moves consist of simple random spin flips. If $E_{o}$ and $E_{n}$ are used to denote the energy before and after a trial move, respectively, the acceptance criteria for such a move can be expressed as

$$
A(o \rightarrow n)=\min \left[1, \frac{g\left(E_{o}\right)}{g\left(E_{n}\right)}\right] .
$$

At the beginning of a simulation, Wang and Landau recommended that the range $\left[E_{\min }, E_{\max }\right]$ be broken up into several smaller, overlapping energy windows. Independent simulations are then conducted in each window. For each window, an energy histogram $H(E)$ is constructed and updated throughout the simulation. Every time that an energy level $E$ is visited, $H(E)$ is updated according to $H(E) \rightarrow H(E)+1$, and $g(E)$ is updated according to $g(E) \rightarrow g(E) \times f$, where $f$ is an arbitrary convergence factor. This factor must be greater than unity; its function will become clear later. Wang and Landau recommended an initial value of $\ln f=1$.

The process outlined here is continued until $H(E)$ becomes sufficiently flat. In this work, "flatness" is considered to be attained when $H(E)$ for all $E$ in a given energy window is within $20 \%$ of the average histogram for that window, $\langle H(E)\rangle$. When this condition is satisfied, a smaller convergence factor $f_{i}$ is adopted according to some arbitrary rule, e.g., $f_{i}=\sqrt{f_{i-1}}$. Any function that monotonically decreases $f$ to unity can be used to reduce the value of $f$. The histogram entries are set to zero, and a new simulation cycle is initiated. The process is repeated until $f \rightarrow 1$, at which point the density of states is not changing in a significant manner, and convergence to a final, "true" value is achieved.

It is important to emphasize the fact that detailed balance is not obeyed in the DOS method, particularly in the initial stages of the simulation, because the density of states is being continuously updated. In the final stages of simulation, however, as $f \rightarrow 1$ the density of states converges to its "true" value. From Eq. (1) we have

$$
\frac{1}{g\left(E_{o}\right)} K(o \rightarrow n)=\frac{1}{g\left(E_{n}\right)} K(n \rightarrow o),
$$

where $1 / g\left(E_{o}\right)$ is the probability of being at energy $E_{o}$, and $K(o \rightarrow n)$ is the probability for a transition from $E_{o}$ to $E_{n}$. Detailed balance is therefore satisfied with accuracy proportional to $\ln f$.

The energy surface of a molecular system is more complicated than that of an Ising system, and more elaborate trial moves are often required for their simulation. In the particular case of polymeric molecules, local moves such as kinkjump, crankshaft, and end-rotation ${ }^{10}$ can be used to sample configuration space. These trial moves, however, are fairly localized, and in fact they mimic the actual motion of the chain at the scale of individual segments. Nonlocal moves, such as reptation, are necessary to achieve molecular relaxation over longer length scales. ${ }^{11}$

At low temperatures or elevated densities, conventional "polymer-simulation" trials moves are generally insufficient. In those situations, biased moves provide one of the only viable means of exploring configuration space with reasonable efficiency. ${ }^{12}$ The question then becomes how to incorporate these biased moves in the context of DOS sampling. To propose our algorithm, we use the fact that biased trial moves rely on the idea that the function $K(o \rightarrow n)$ [see Eq. (2)] can be factored into the probability of proposing a transition form $o$ to $n, \mathcal{T}(o \rightarrow n)$ and the probability of accepting the proposed transition, $A(o \rightarrow n)$, i.e.,

$$
K(o \rightarrow n)=\mathcal{T}(o \rightarrow n) A(o \rightarrow n)
$$

and the acceptance criteria take the form

$$
A(o \rightarrow n)=\min \left[1, \frac{g\left(E_{o}\right) \mathcal{T}(n \rightarrow o)}{g\left(E_{n}\right) \mathcal{T}(o \rightarrow n)}\right] .
$$

The particular form of the function $\mathcal{T}(o \rightarrow n)$ depends on the details of the trial move. In this work, we use simple configurational bias $^{13,14}$ and topological configurational bias ${ }^{15}$ moves. Simple configurational bias moves involve cutting and regrowing $n$ end sites of the chains, one at a time, with a bias designed to avoid overlaps. At each step $i$ of the growth process, all possible trial orientations $k$ of a segment $(k=6$ on a cubic lattice) are considered, and one position $j$ is chosen from the $e_{i}$ "empty" or available trial positions with uniform probability, i.e.,

$$
p_{i}^{j}=\frac{1}{e_{i}} .
$$

The so-called normalized Rosenbluth weight of the chain is constructed according to

$$
R_{W}^{F}=\prod_{i=1}^{n} e_{i}
$$

which can be thought of as the usual Rosenbluth weight ${ }^{13}$ in the limit $\beta \rightarrow 0$, where $\beta=1 / k_{B} T$. The Rosenbluth weight for the reverse move is reconstructed by calculating the number of empty trial positions available to each segment as the chain is grown into its original configuration. The acceptance criteria for this move are therefore given by

$$
A(o \rightarrow n)=\min \left[1, \frac{g\left(E_{o}\right) R_{W}^{F}}{g\left(E_{n}\right) R_{W}^{R}}\right],
$$


where the superscripts $F$ and $R$ refer to the forward and reverse moves, respectively.

In the case of a topological configurational bias move, an inner section of a chain molecule is cut and regrown in such a way as to satisfy the connectivity constraints of the molecule. The bias is dictated by the number of empty trial sites $e_{i}$ accessible to each growing segment, and also by the number of random walks $N_{R W}^{i}(l)$ that exist between trial position $l$ to the point where the chain was originally cut. The direction $j$ of the current regrowing site $i$ is chosen from the probability distribution

$$
p_{i}^{j}=\frac{N_{R W}^{i}(j)}{\sum_{l=1}^{e_{i}} N_{R W}^{i}(l)} .
$$

The Rosenbluth weight $R_{W}^{F}$ and the random walk weight $G_{W}^{F}$ for a trial move are given by

$$
\begin{aligned}
R_{W}^{F} & =\prod_{i=1}^{n} \sum_{l=1}^{e_{i}} N_{R W}^{i}(l), \\
G_{W}^{F} & =\prod_{i=1}^{n} N_{R W}^{i}(j) .
\end{aligned}
$$

The Rosenbluth and the random walk weights for the reverse move are calculated in a similar manner; the acceptance criteria for this type of move are given by

$$
A(o \rightarrow n)=\min \left[1, \frac{g\left(E_{o}\right) R_{W}^{F} G_{W}^{R}}{g\left(E_{n}\right) R_{W}^{R} G_{W}^{F}}\right] .
$$

We refer to this algorithm configurational bias density of states Monte Carlo (CBDOS).

The biased sampling ideas discussed above can also be extended to reptation, end-rotation, and crankshaft moves. In order to distinguish these from the traditional, random moves, we will refer to them as biased reptation and biased local moves.

It is important to note that the algorithm described above only gives $g(E)$ to within an arbitrary multiplicative factor. To recover absolute values, $g(E)$ can be determined for a state for which thermodynamic properties are known (e.g., ideal gas). Having generated $g(E)$ to within the required accuracy, other properties of the system, such as the thermodynamic internal energy $\langle E\rangle$, can be calculated using standard statistical mechanics procedures.

$$
\langle E\rangle=\frac{\Sigma_{E} E g(E) \exp (-\beta E)}{\sum_{E} g(E) \exp (-\beta E)} .
$$

The free energy and the entropy can be calculated according to

$$
F^{*}=\frac{F}{k_{B} T}=-\ln \left[\sum_{E} g(E) \exp (-\beta E)\right]
$$

and

$$
S^{*}=\frac{S}{k_{B}}=\frac{\langle E\rangle-F}{k_{B} T} .
$$

\section{B. Model and simulation details}

Polymer chains are represented by self-avoiding random walks on a square lattice of unit $\sigma$. Sites along the chains interact via a nearest-neighbor potential energy of magnitude $-\varepsilon$. The chain length $L$ considered here is 100 sites. The number of chains $N$ used in this work is 125 . The typical thickness of the film is $\approx 23$ lattice units and the dimensions of the simulation box in the directions perpendicular to the thickness of the film are 25 lattice units. All results are reported in reduced variables, i.e., temperature $T^{*}=k T / \epsilon$ and density $\phi=N \sigma^{3} / V$.

In addition to the simulations using the DOS method, we performed independent simulations in the canonical ensemble in order to verify the results from the DOS method. The starting point for the simulations was the spontaneous formation of a thick, free-standing film. The initial temperature and density were $T^{*}=2.2$ and $\phi=0.85$, respectively. In the case of canonical simulations, each simulation was run until the system was well equilibrated. The temperature was lowered in a stepwise manner. The final, fully relaxed configuration for a given temperature was subsequently taken as the starting point for the next run at a slightly lower temperature. At each temperature, the displacement of the center of mass of the chains $\left[d_{1}(t)\right]$ and the site displacement in the center of mass coordinates $\left[d_{2}(t)\right]$ were monitored. The simulations were run long enough to ensure that $d_{1}(t)$ was several radii of gyration, and that $d_{2}(t)$ was constant. The trial moves discussed in the context of the DOS method were used in the case of canonical simulations as well. An apparent glass transition temperature was estimated by measuring the thickness of a given film as a function of temperature. The point at which the slope of the thickness versus temperature graph changes was identified with $T_{g}$.

In the case of the CBDOS simulations, the initial configuration generated was immediately quenched at a range of different temperatures to obtain starting configurations with different energies over the energy range of interest. A histogram of film thickness, $h(E)$, was maintained as a function of energy in order to calculate the average thickness as a function of temperature.

In order to verify the validity of the proposed CBDOS algorithm, several properties of the system were determined as a function of temperature and compared to results from conventional canonical-ensemble simulations. To do this, we calculated $g(E)$ over a range of overlapping energy windows spanning a temperature range from 2.0 to 1.0. The typical size of the energy windows was $1000 \varepsilon$. The overlap region between neighboring windows was $300 \varepsilon$.

To examine the efficiency of different trial moves in converging towards the true $g(E)$, we performed extensive simulations in two energy windows; one corresponding to the high temperature melt state (corresponding to $T^{*} \approx 1.9$ ) and the second one in an energy range corresponding to the vicinity of the glass transition (i.e., $\left.T^{*} \approx 1.0\right){ }^{12}$ Since $f$ is a measure of the convergence of $g(E)$ to the true value, we kept track of $f$ as a function of number of Monte Carlo steps (MCS). A MCS was defined as an attempted move for each occupied site in the system. The typical densities were $\phi=0.8$ in the high-energy region and $\phi=0.97$ in the low- 


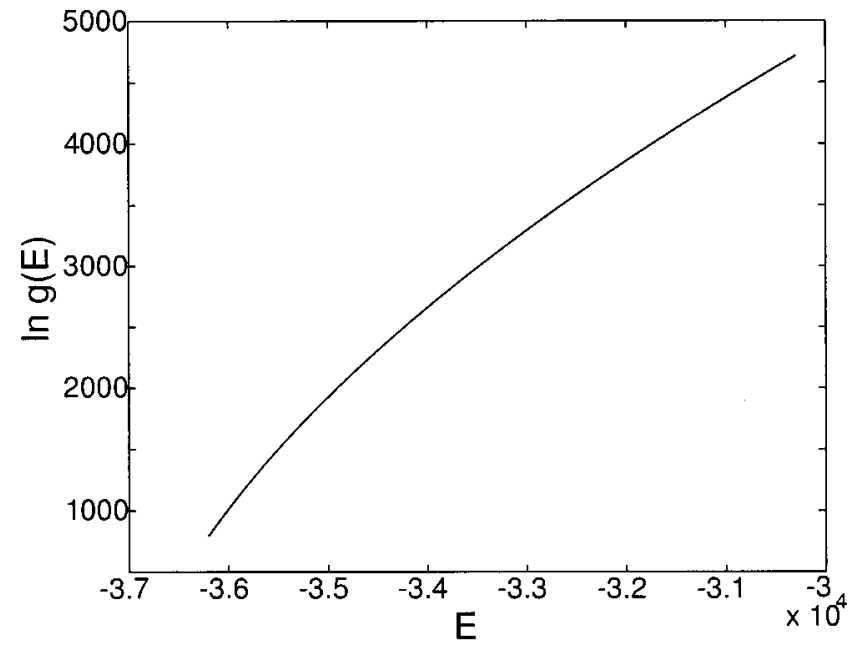

FIG. 1. Density of states as a function of energy.

energy region. The different moves used were random local moves, biased local moves, random reptation, biased reptation, and configurational bias moves.

We also calculated the end-to-end autocorrelation function $r(t)$ for the chains in two energy windows corresponding to the temperatures of $T^{*} \approx 2.0$ and $T^{*} \approx 1.3$. This was done because $r(t)$ provides a stringent measure of the rate at which new configurations are being generated in a given simulation.

It should be noted that the case of conventional canonical ensemble simulations, one has to wait for an equilibrated configuration from a previous run as a starting configuration for a lower temperature in order to enable the system to relax fully. This is especially true at lower temperatures near the glass transition, where a system might get trapped in a local minimum if it is quenched too rapidly. While this problem can be partially alleviated by using the more elaborate moves mentioned above, at elevated densities (near the glass transition) we still followed the above procedure to make sure that the starting configurations did not retain a "memory" of the higher temperatures.

In the case of CBDOS, the above limitation does not arise due to the athermal nature of the simulation. Neverthe-

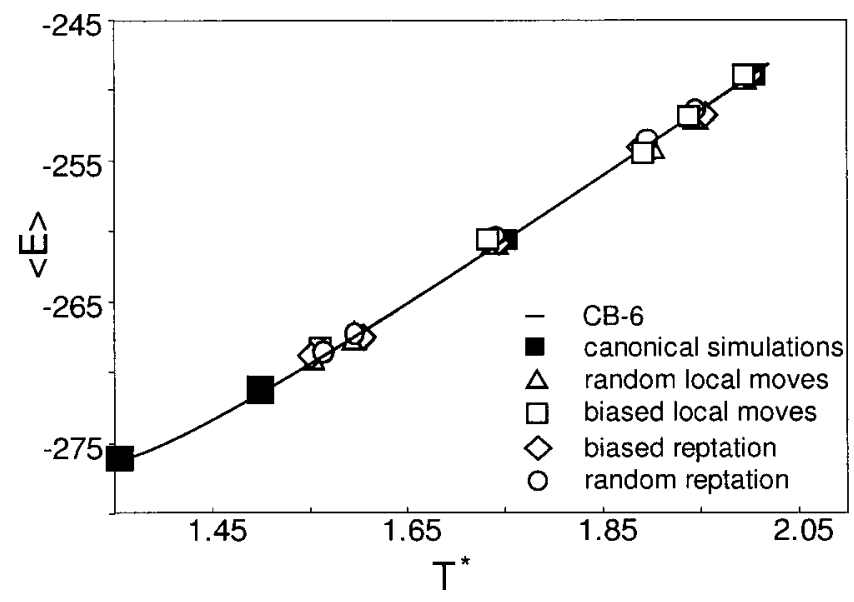

FIG. 2. Comparison of average energy per chain.

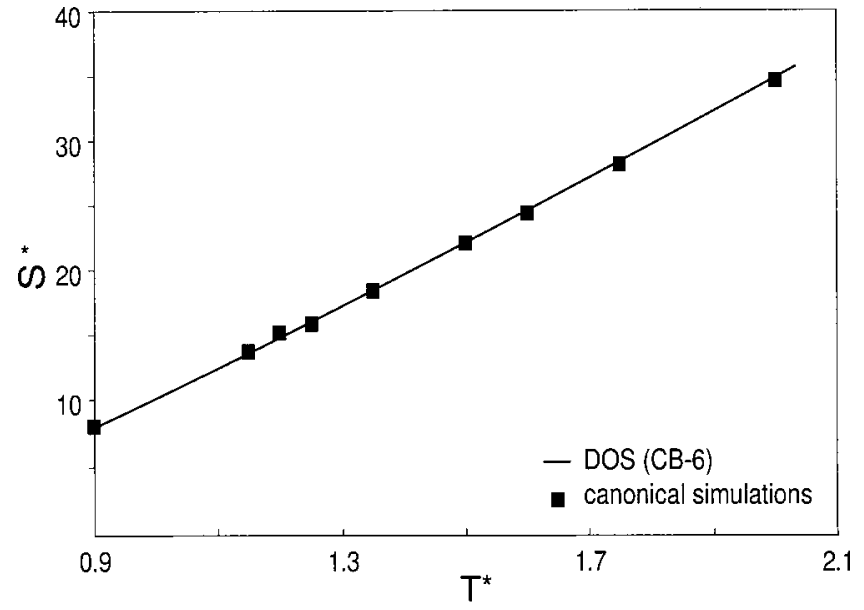

FIG. 3. Comparison of entropy per chain.

less, one has to ensure that the energy window used in a run are wide enough for the system to overcome energy barriers and to sample configuration space efficiently. In the case of canonical simulations, the overall simulation process is essentially serial, and it takes around 4 to 5 weeks of computer time to generate canonical data over the temperature range of interest. By contrast, after the initial quenching required for the different starting energy configurations in the DOS methods, the different windows can be simulated independently and the process requires around 1 week of computer time for temperatures near the glassy state, and 2 to 3 days for temperatures in the regime of the polymer melt.

An additional advantage of the CBDOS method is that it provides a direct estimate of the partition function (to within a multiplicative constant), and hence the free energy and the entropy. In contrast, in the canonical ensemble one has to resort to a method such as incremental chain site insertion (Sec. III) in order to calculate $F$ and $S^{*}$ from Eq. (14).

\section{RESULTS}

Figure 1 shows the calculated density of states as a function of energy. The curves from the different methods all agree with each other and, therefore, we have only shown the curve calculated from CB-6, i.e., configurational bias move with 6 sites that were cut and regrown.

Figure 2 shows results for the average energy $\langle E\rangle$ per chain obtained for different types of moves and from canonical simulations. The agreement between the methods is excellent over the entire temperature range studied. The error bars in all the figures are smaller than the size of the symbols used.

The entropy per chain $S^{*}$ as a function of temperature is plotted in Fig. 3 for both CBDOS and canonical simulations. For the canonical simulations the free energy of the system was calculated from $F=-p V+\mu N,{ }^{16}$ where $\mu$ is the chemical potential of the chain, $p$ is the pressure, and $V$ is the volume of the system. In the case of a free-standing film, the pressure $p=0$. The chemical potential, $\mu$, was calculated by using the incremental insertion method proposed by Kumar and co-workers ${ }^{17}$ and the relation of the excess chemical potential to the Rosenbluth weight of the chain ${ }^{18,19}$ given by 
TABLE I. Comparison of film thickness $h$ from CBDO Sand canonical simulations.

\begin{tabular}{ccc}
\hline \hline$T$ & $h$ CBDOS & $h$ canonical \\
\hline 2.00 & 24.22 & $24.5 \pm 0.5$ \\
1.35 & 21.16 & $21.5 \pm 0.5$ \\
1.20 & 20.85 & $20.8 \pm 0.4$ \\
\hline \hline
\end{tabular}

$$
-\beta \mu_{s}^{\mathrm{ex}}=\ln \left\langle\frac{R_{W s}}{k^{s}}\right\rangle
$$

and

$$
\mu^{\mathrm{ex}}=\frac{L}{S} \mu_{s}^{\mathrm{ex}}
$$

where $\mu_{s}^{\mathrm{ex}}$ and $R_{W s}$ are the excess chemical potential and the Rosenbluth weight for $s$ sites in the chain, respectively, $\mu^{\text {ex }}$ is the excess chemical potential for the entire chain, and $\langle\ldots\rangle$ denotes an ensemble average. This method can be thought of as a modified Widom method ${ }^{20}$ in which $s$ sites are inserted at the end of the chain and the insertions are biased by their Boltzmann factors in order to prevent steric overlaps and get better statistics. Equation (14) was then used to calculate the entropy. Since CBDOS provides $S^{*}$ to within a constant, the CBDOS results have been offset by a constant in order to achieve overlap with the results from canonical simulations.

The system considered in this work is a free-standing film; its thickness $h$ as a function of temperature is also a property of interest. The film thickness was periodically evaluated by calculating the center of mass of the film; the "bulk" density was determined from the mean density in the region of the film near the center of mass. The film thickness was calculated by counting the number of lattice planes parallel to the film having density greater than half the "bulk" density. Table I lists the values of $h$ for three temperatures. As one can see, the agreement of the values from the CBDOS method and those from canonical simulations is excellent.

The specific heat $C_{v}$ depends on fluctuations of energy, and is therefore particularly sensitive to errors in the calculation of $g(E)$. It can be determined from

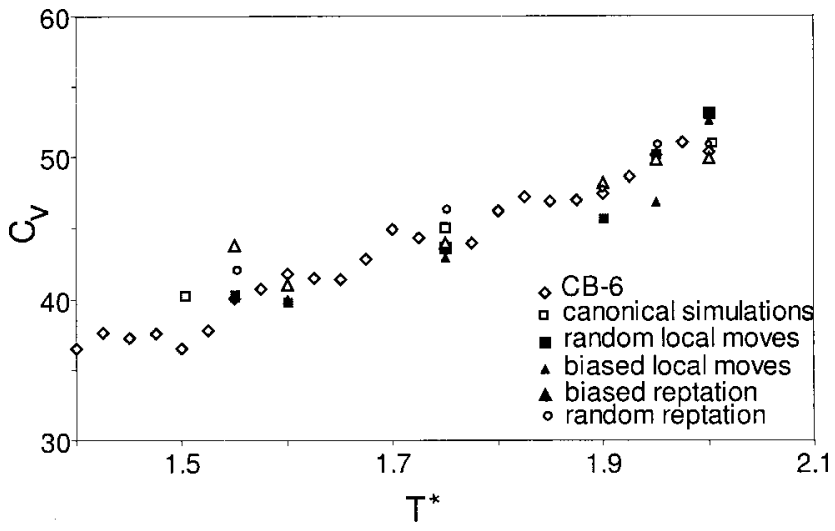

FIG. 4. Comparison of specific heat per chain.

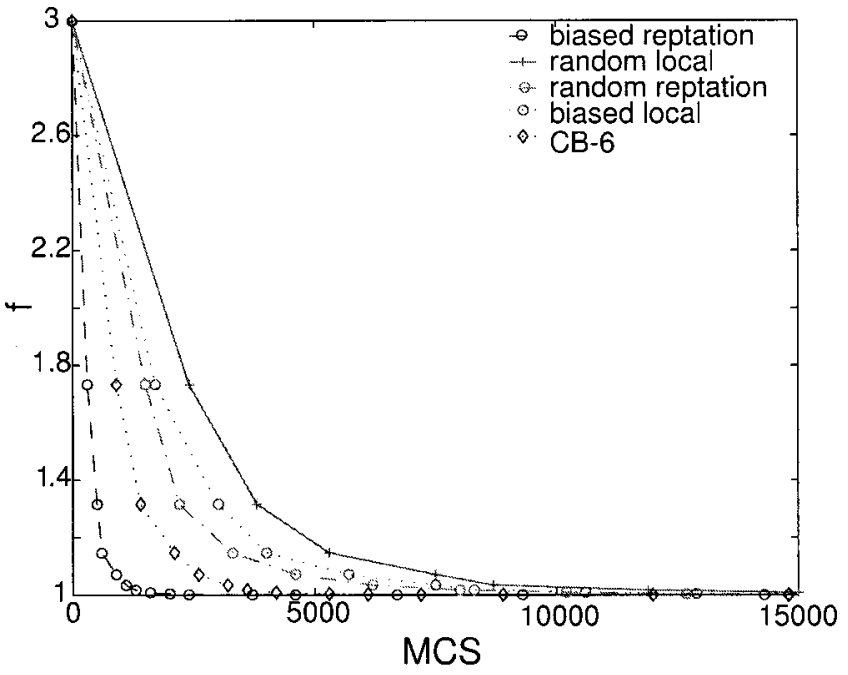

FIG. 5. Convergence factor $f$ (see text) as a function of number of Monte Carlo steps for the high-energy (low-density) window.

$$
C_{v}=\frac{\left\langle(E-\langle E\rangle)^{2}\right\rangle}{N k T^{2}} .
$$

The specific heat obtained from the different types of moves employed and from fluctuations in the canonical ensemble (Fig. 4) agree closely with each other over a wide range of temperature.

For the high-energy window (Fig. 5, $T^{*} \approx 1.9$ ), we have plotted the convergence factor $f$ as a function of number of Monte Carlo steps for three different kinds of moves, namely local, reptation, and configurational bias with six segments (CB-6). As one can see, biased reptation moves require the smallest number of Monte Carlo steps to reach a given value of $f$. This is closely followed by CB-6. As expected, the biased versions of the local moves and reptation also converge faster than simple random displacements. In contrast, near the apparent glass transition (i.e., in the low-energy window, $T^{*} \approx 1.0$ ) (Fig. 6), both the simple and biased versions

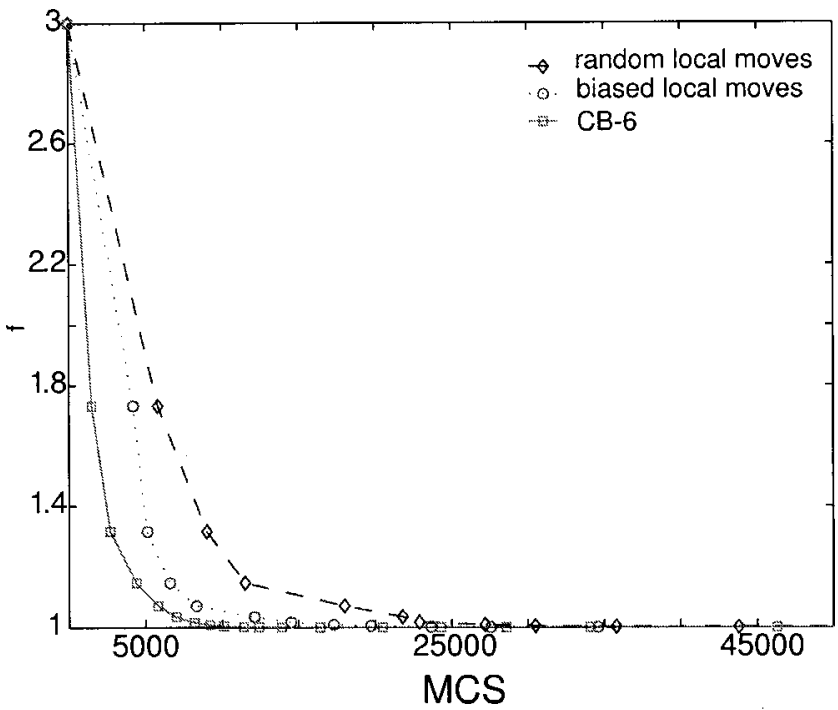

FIG. 6. Convergence factor $f$ (see text) as a function of number of Monte Carlo steps for the low-energy (high-density) window. 


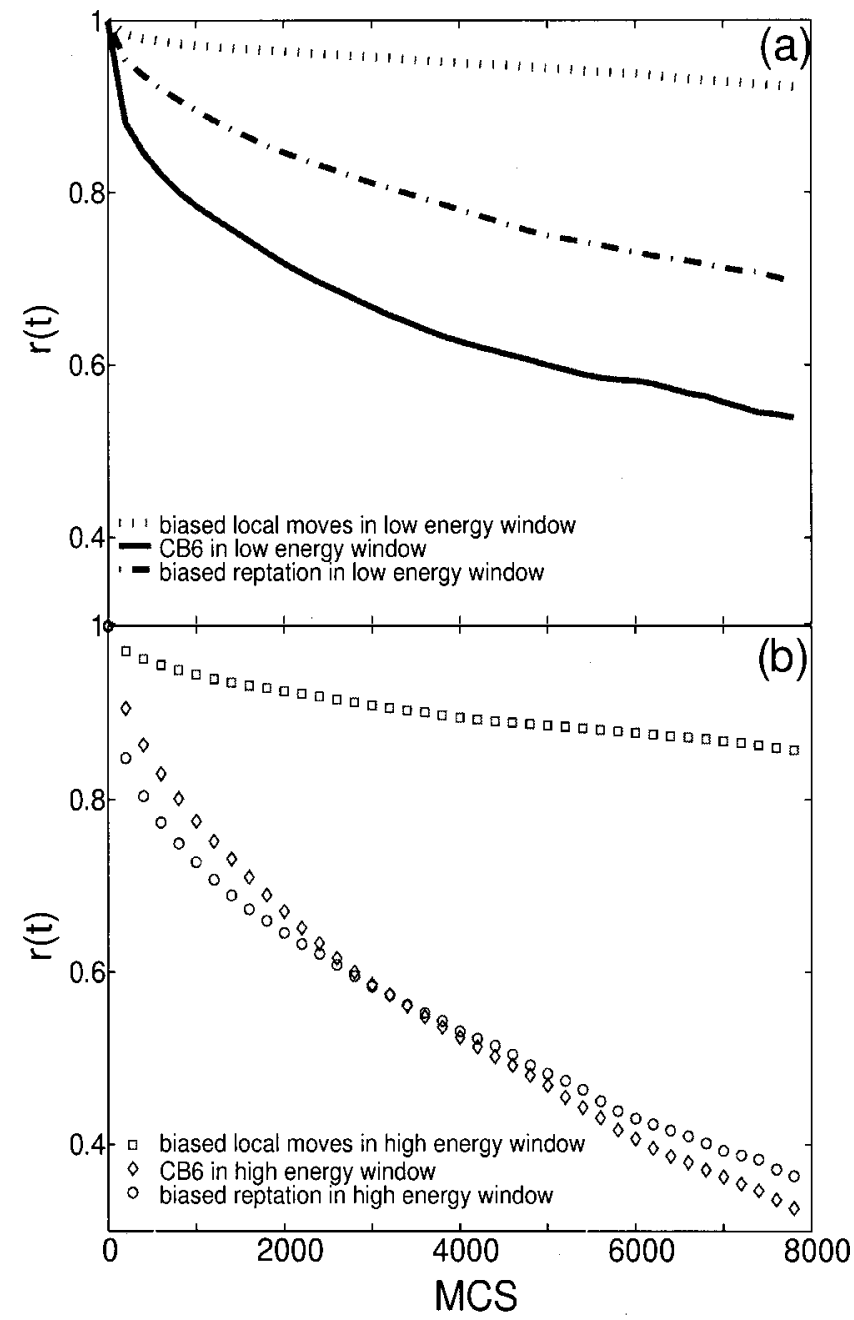

FIG. 7. End-to-end correlation function for different energy windows, (a) low-energy (high density, $T^{*} \approx 1.3$ ) and (b) high-energy (low density, $T^{*} \approx 2.0$ ) window.

of reptation failed to converge. Only the CB-6 moves was effective in this region, followed by biased and usual random moves, respectively.

An end-to-end autocorrelation function $r(t)$ for the chain is defined according to

$$
r(t)=\frac{\langle\mathbf{R}(t) \cdot \mathbf{R}(0)\rangle}{\langle\mathbf{R}(0) \cdot \mathbf{R}(0)\rangle},
$$

where $\mathbf{R}(t)$ is the end-end-end vector at time $t$. As one can see from Fig. 7 , in the high-energy (low-density, $T^{*} \approx 2.0$ ) window, biased reptation is the fastest followed by CB-6 and biased local moves, respectively. However, the in the lowenergy (high-density, $T^{*} \approx 1.3$ ) window, CB-6 provides the fastest relaxation of the chain followed by biased reptation and biased local moves, respectively. This further corroborates the fact that advanced moves are necessary in order to sample the energy space efficiently at high densities. At low densities, the chain is able to reptate freely and this leads to fast relaxation. At high densities the chain is unable to relax if the terminal sites are blocked; the resulting end-to-end relaxation of the chain becomes slow.

\section{CONCLUSIONS}

We have demonstrated the use of biased, polymer specific trial moves such as configurational bias in the context of a density-of-states based sampling method. We have shown that it gives results consistent with those of completely independent canonical simulations. The agreement is excellent both for single chain quantities as well as for large scale properties of the film (e.g., film thickness $h$ ).

We have compared the efficiency of various types of commonly used trial moves for polymers. We found that reptation is highly effective at low densities, but it fails to converge (within the time scale of our simulations) at elevated densities, where more elaborate techniques are necessary to arrive at the correct density of states $g(E)$.

The proposed method appears to be highly effective (in terms of computing time) compared to the more conventional canonical simulation technique. The absence of temperature in density-of-states sampling methods is helpful in overcoming the inherently slow dynamics (or high energy barriers) that characterize near glassy systems at low temperatures.

\section{ACKNOWLEDGMENTS}

This work is supported by the Division of Chemical Sciences, Office of Science, and the U.S. Department of Energy (DE-FG02-99ER14961). Support from the Semiconductor Research Corporation is also acknowledged.

${ }^{1}$ J. L. Keddie, R. A. L. Jones, and R. Cory, Europhys. Lett. 27, 59 (1994).

${ }^{2}$ E. Dalnoki-Veess, J. A. Forrest, C. Murray, C. Gigault, and J. R. Dutcher, Phys. Rev. E 63, 031801 (2001).

${ }^{3}$ D. S. Fryer, P. F. Nealey, and J. J. de Pablo, Macromolecules 33, 6439 (2000).

${ }^{4}$ J. A. Torres, P. F. Nealey, and J. J. de Pablo, Phys. Rev. Lett. 85, 3221 (2000).

${ }^{5}$ D. S. Fryer, R. D. Peters, E. J. Kim, J. E. Tomaszewski, J. J. de Pablo, P. F. Nealey, C. C. White, and W. L. Wu, Macromolecules 34, 5627 (2001).

${ }^{6}$ F. H. Stillinger and T. A. Weber, Phys. Rev. A 28, 2408 (1983).

${ }^{7}$ M. Goldstein, J. Chem. Phys. 51, 3728 (1969).

${ }^{8}$ F. Wang and D. Landau, Phys. Rev. Lett. 86, 2050 (2001)

${ }^{9}$ F. Wang and D. Landau, Phys. Rev. E 64, 056101 (2001).

${ }^{10}$ M. Lax and K. Brender, J. Chem. Phys. 67, 1785 (1977).

${ }^{11}$ F. Mandel, J. Chem. Phys. 70, 3984 (1979).

${ }^{12}$ T. Jain and J. J. dePablo, Macromolecules 35, 2167 (2002).

${ }^{13}$ J. dePablo, M. Laso, and U. Suter, J. Chem. Phys. 96, 2395 (1992).

${ }^{14}$ J. Siepmann and D. Frenkel, Mol. Phys. 75, 59 (1992).

${ }^{15}$ M. Dijsktra, D. Frenkel, and J. Hansen, J. Chem. Phys. 101, 3179 (1994).

${ }^{16}$ In the case of a system with interfaces, $F=-p V+\mu N+\gamma A$, where $A$ is the interfacial area of the system and $\gamma$ is the surface tension. In our system we found that the contribution of the $\gamma A$ term is small compared to $\mu N$ and only slightly affects the entropy as calculated from Eq. (14). Therefore, for the sake of simplicity, we have ignored this term.

${ }^{17}$ S. Kumar, I. Szleifer, and A. Panagiotopoiulos, Phys. Rev. Lett. 66, 2935 (1991).

${ }^{18}$ J. J. dePablo, Q. L. Yan, and F. A. Escobedo, Annu. Rev. Phys. Chem. 50, 377 (1199).

${ }^{19}$ D. Frenkel and B. Smit, Understanding Molecular Simulation (Academic, New York, 1996).

${ }^{20}$ B. Widom, J. Chem. Phys. 39, 2802 (1963). 